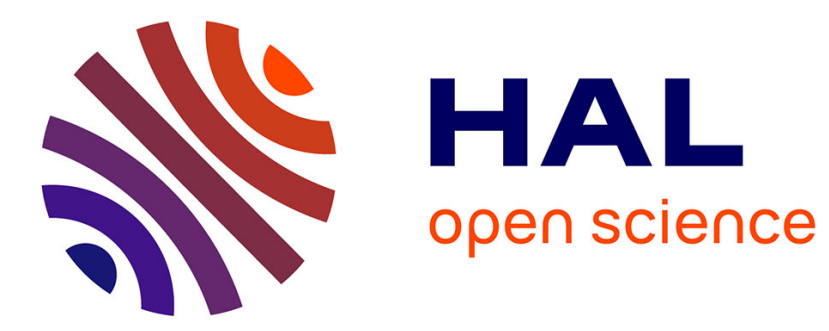

\title{
Exact diffusion constant for one-dimensional asymmetric exclusion models
}

\author{
Bernard Derrida, M. R. Evans, D. Mukamel
}

\section{To cite this version:}

Bernard Derrida, M. R. Evans, D. Mukamel. Exact diffusion constant for one-dimensional asymmetric exclusion models. Journal of Physics A: Mathematical and General (1975 - 2006), 1993, 26 (19), pp.4911-4918. 10.1088/0305-4470/26/19/023 . hal-03282963

\section{HAL Id: hal-03282963 \\ https://hal.science/hal-03282963}

Submitted on 19 Jul 2021

HAL is a multi-disciplinary open access archive for the deposit and dissemination of scientific research documents, whether they are published or not. The documents may come from teaching and research institutions in France or abroad, or from public or private research centers.
L'archive ouverte pluridisciplinaire HAL, est destinée au dépôt et à la diffusion de documents scientifiques de niveau recherche, publiés ou non, émanant des établissements d'enseignement et de recherche français ou étrangers, des laboratoires publics ou privés. 


\title{
Exact diffusion constant for one-dimensional asymmetric exclusion models
}

\author{
B Derrida†, M R Evans $\nmid$ and D Mukamelł \\ $\dagger$ Service de Physique Theorique, CE Saclay, F-91191 Gif-sur-Yvette Cedex, France \\ $\ddagger$ Department of Physics, Weizmann Institute, Rehovot 76100, Israel
}

Received 29 April 1993

\begin{abstract}
The one-dimensional fully asymmetric exclusion model, which describes a system of particles hopping in a preferred direction with hard core interactions, is considered on a ring of size $N$. The steady state of this system is known (all configurations have equal weight), which allows for easy computation of the average velocity of a particle in the steady state. Here an exact expression for the diffusion constant of a particle is obtained for arbitrary number of particles and system size, by using a matrix formulation. Two limits of infinite system size $N$ are discussed: frstly, when the number of particles remains finite as $N \rightarrow \infty$ the diffusion constant remains dependent on the exact number of particles due to correlations between successive collisions; secondly, when the density $\rho$ of particles is non-zero (i.e. when the number of particles is equal to $N \rho$ as $N \rightarrow \infty$ ) the diffusion constant scales as $N^{-1 / 2}$. The exponent $-1 / 2$ is related to the dynamic exponent $z=3 / 2$ of the KPZ equation in $(1+1)$ dimensions.
\end{abstract}

Particles hopping in a preferred direction with stochastic dynamics and hard core interactions are simple examples of non-equilibrium systems [1]. Physically they are models of driven lattice gases [2] or hopping conductivity [3] and are closely related to growth processes [4,5]. A particularly simple example of a driven lattice gas is the one-dimensional asymmetric exclusion process [6]. The model describes particles which jump independently to their right with hard core repulsion along a one-dimensional lattice. It is one of the few non-equilibrium models for which the steady state is exactly known and all equal-time correlation functions can be calculated [7-11]. For periodic boundary conditions all configurations have equal weight in the steady state [7]. The case of open boundary conditions [5,12], where particles enter at one end of the lattice and are removed at the opposite end, is more complicated, nevertheless the steady state can still be solved exactly [8-11]. It was shown recently [11] that a simple way of representing the steady state is to write the weights of configurations as products of non-commuting matrices. This approach makes the exact solution much easier to obtain and has been extended to other situations such as a mixture of two species of particles on a ring [13]. Although it holds the promise of wider application, the matrix approach has so far only been used to obtain steady-state, equal-time correlation functions.

The time dependence of these models at present appears more difficult, although it has been shown that for periodic boundary conditions the eigenvalues of the master equation can be computed via the Bethe ansatz [14-16]. Of further interest is the behaviour of correlations in time. A quantity related to such correlations is the diffusion constant of a marked particle. It is known that, when an average is carried out over random initial conditions for an infinite system, the long time behaviour of the variance of the distance 
travelled by a marked particle is proportional to $t$, the constant of proportionality being the diffusion constant [17-19].

On the other hand when the initial configuration is fixed the variance of the distance travelled is expected to increase as $t^{2 / 3}[20,21]$. Here we consider a finite system of size $N$ in the long time limit $(t \rightarrow \infty)$. Using a matrix formulation inspired by [11,13], we obtain an explicit expression for the diffusion constant for all lattice sizes and all possible densities of particles. Because we take the limit $t \rightarrow \infty$ at finite $N$ the value of the diffusion constant does not depend on initial conditions.

Let us first define the one-dimensional asymmetric exclusion model that we consider. Each site $i$ ( $1 \leqslant i \leqslant N$ ) of a one-dimensional lattice of $N$ sites is either occupied by a particle $\left(\tau_{i}=1\right)$ or empty $\left(\tau_{i}=0\right)$. The lattice has periodic boundary conditions $\left(\tau_{N+i}=\tau_{i}\right)$. The continuous time dynamics are as follows. During any infinitesimal time interval $\mathrm{d} t$ each particle has a probability $\mathrm{d} t$ of jumping to its right-hand neighbour if this neighbouring site is empty.

The number of particles on the lattice is fixed to be $M+1$ which reflects the fact that one of the particles, without loss of generality the first, is marked (although this does not in any way affect its dynamics). We denote by $Y_{t}$ the number of hops the marked particle has made up to time $t$, where $Y_{0}=0$. A configuration $C$ of the system specifies the positions of the $M$ unmarked particles relative to the marked one, that is, we make the convention that at time zero the marked particle is at site one and each time the marked particle moves we use the translational invariance of the lattice to relabel the sites so that the position of the marked particle remains site one.

In the long-time limit, the system reaches a steady state where the probabilities $p\left(\tau_{2}, \tau_{3}, \cdots \tau_{N}\right)$ of each configuration are stationary. It is easy to convince oneself that the fact that the number of possible ways of entering and leaving a configuration during time $d t$ are equal (i.e the number of pairs of sites $i, i+1$ with $\tau_{i}=1, \tau_{i+1}=0$ equals the number of pairs with $\tau_{i}=0, \tau_{i+1}=1$ ) implies that any configuration with the correct number of particles has equal probability in the steady state [7]. Thus

$$
p(\mathcal{C})=\left[\left(\begin{array}{c}
N-1 \\
M
\end{array}\right)\right]^{-1}
$$

Our aim is to determine the fluctuations in the distance travelled by the marked particle. For large times, we expect that the average distance travelled is given by

$$
\left\langle Y_{t}\right\rangle \simeq v t
$$

where the velocity $v$ is the rate at which, in the steady state, the marked particle makes hops forward. The form of the steady-state probabilities (1) implies that

$$
v=(N-M-1) /(N-1) \text {. }
$$

One also expects that in the long-time limit

$$
\left\langle Y_{t}^{2}\right\rangle-\left\langle Y_{t}\right\rangle^{2} \simeq \Delta t
$$

where $\Delta$ is the diffusion constant of the marked particle. In contrast to $v$ the diffusion constant $\Delta$ is a non-trivial quantity because it reflects non-equal-time correlations. The main result of this work is the following exact expression for $\Delta$ (for $M+1$ particles on a lattice of $N$ sites):

$$
\Delta=\left(\begin{array}{c}
2 N-3 \\
2 M
\end{array}\right)\left[\left(\begin{array}{c}
N-1 \\
M
\end{array}\right)\right]^{-2}(2 M+1)^{-1}
$$


Before coming to the derivation of (5), let us discuss briefly two limits of interest. First we consider an infinite system containing a finite number of particles. By letting $N \rightarrow \infty$ in (5) with $M$ fixed we obtain

$$
\Delta \rightarrow 4^{M} \frac{(M !)^{2}}{(2 M+1) !} .
$$

In this limit one might believe that the infinite size of the system renders the events of particles encountering each other so rare that the diffusion constant reduces to the value for a collisionless system. However, on inspecting (6) one sees that for all $M \geqslant 1$ the diffusion constant differs from unity, the value it would take if the marked particle were the only particle in the system (i.e. $M=0$ ), and depends on the exact number of particles. The explanation is that once a particle collides with another particle many collisions ensue, therefore the collisions are highly correlated in time.

A second limit we consider is that of a finite density of particles $\rho$ in an infinite system. On taking $N \rightarrow \infty$ in (5) with $\dot{\rho}$ fixed, where

$$
M=N \rho
$$

one obtains to leading order in $N$

$$
\Delta \simeq \frac{\sqrt{\pi}}{2} \frac{(1-\rho)^{3 / 2}}{\rho^{1 / 2}} \frac{1}{N^{1 / 2}}
$$

The exponent $\frac{1}{2}$ can be related to the dynamic exponent of the KPZ equation [22] in $1+1$ dimensions as follows. There exists a mapping from the asymmetric exclusion process to $(1+1)$-dimensional single step growth models in which the movement of a particle is equated with the growth of the surface height at a site by one unit [7]. From (8) the variance of the surface heights at time $t$, or surface roughness, scales as $\left[\left\langle Y_{t}^{2}\right\rangle-\left\langle Y_{t}\right\rangle^{2}\right] \sim N^{-1 / 2} t$. However, for a growth process with linear size $N$ of the substrate, the roughness cannot increase indefinitely and can only grow to $O(N)$. The time scale at which the crossover occurs, so that the interface appears rough on the scale of the system size, is $N^{3 / 2}$. The exponent $\frac{3}{2}$ is equivalent to $z$ the dynamic exponent of the KPZ equation $[22,4]$.

We now outline a derivation of (5). Let us define $P_{t}(C ; Y)$ as the probability at time $t$ of finding the system in configuration $\mathcal{C}$ and of the marked particle having made exactly $Y$ moves, averaged over any ensemble of initial configurations. The time evolution of $P_{t}(\mathcal{C} ; Y)$ is governed by the master equation

$$
\begin{aligned}
\frac{\partial}{\partial t} P_{t}(\mathcal{C} ; Y)= & \sum_{\mathcal{C}^{\prime}}\left[K_{0}\left(\mathcal{C}, \mathcal{C}^{\prime}\right) P_{t}\left(\mathcal{C}^{\prime} ; Y\right)+K_{1}\left(\mathcal{C}, \mathcal{C}^{\prime}\right) P_{t}\left(\mathcal{C}^{\prime} ; Y-1\right)\right] \\
& -\sum_{\mathcal{C}^{\prime}}\left[K_{0}\left(\mathcal{C}^{\prime}, \mathcal{C}\right)+K_{1}\left(\mathcal{C}^{\prime}, \mathcal{C}\right)\right] P_{t}(\mathcal{C} ; Y)
\end{aligned}
$$

where $K_{0}\left(\mathcal{C}, \mathcal{C}^{\prime}\right)$ is the rate of transition from configuration $\mathcal{C}^{\prime}$ to $\mathcal{C}$ by moving an unmarked particle and $K_{1}\left(\mathcal{C}, \mathcal{C}^{\prime}\right)$ is the rate of transition from configuration $\mathcal{C}^{\prime}$ to $\mathcal{C}$ by moving the marked particle.

Let us also consider $p_{t}(\mathcal{C}), q_{t}(\mathcal{C})$ defined by

$$
p_{t}(\mathcal{C})=\sum_{Y} P_{t}(\mathcal{C} ; Y) \quad q_{t}(\mathcal{C})=\sum_{Y} Y P_{t}(\mathcal{C} ; Y) .
$$

Thus $p_{t}(\mathcal{C})$ is the probability that the system is in configuration $\mathcal{C}$ at time $t$ and $q_{t}(\mathcal{C}) / p_{t}(\mathcal{C})$ is the average distance travelled by the marked particle given that the system is in configuration 
$\mathcal{C}$ at time $t$. These quantities satisfy

$$
\begin{aligned}
\frac{\partial}{\partial t} p_{t}(\mathcal{C})=\sum_{\mathcal{C}^{\prime}} & {\left[K_{0}\left(\mathcal{C}, \mathcal{C}^{\prime}\right)+K_{1}\left(\mathcal{C}, \mathcal{C}^{\prime}\right)\right] p_{t}\left(\mathcal{C}^{\prime}\right)-\sum_{\mathcal{C}^{\prime}}\left[K_{0}\left(\mathcal{C}^{\prime}, \mathcal{C}\right)+K_{1}\left(\mathcal{C}^{\prime}, \mathcal{C}\right)\right] p_{t}(\mathcal{C}) } \\
\frac{\partial}{\partial t} q_{t}(\mathcal{C})=\sum_{\mathcal{C}^{\prime}} & {\left[K_{0}\left(\mathcal{C}, \mathcal{C}^{\prime}\right)+K_{1}\left(\mathcal{C}, \mathcal{C}^{\prime}\right)\right] q_{t}\left(\mathcal{C}^{\prime}\right)-\sum_{\mathcal{C}^{\prime}}\left[K_{0}\left(\mathcal{C}^{\prime}, \mathcal{C}\right)+K_{1}\left(\mathcal{C}^{\prime}, \mathcal{C}\right)\right] q_{t}(\mathcal{C}) } \\
& +\sum_{\mathcal{C}^{\prime}} K_{1}\left(\mathcal{C}, \mathcal{C}^{\prime}\right) p_{t}\left(\mathcal{C}^{\prime}\right) .
\end{aligned}
$$

The time derivatives of the first two moments of $Y_{t}$ may be expressed in terms of these quantities as

$$
\begin{aligned}
\frac{\partial}{\partial t}\left\langle Y_{t}\right\rangle & =\frac{\partial}{\partial t} \sum_{\mathcal{C}} q_{t}(\mathcal{C})=\sum_{\mathcal{C}, \mathcal{C}^{\prime}} K_{1}\left(\mathcal{C}, \mathcal{C}^{\prime}\right) p_{t}\left(\mathcal{C}^{\prime}\right) \\
\frac{\partial}{\partial t}\left\langle Y_{t}^{2}\right\rangle & =\frac{\partial}{\partial t} \sum_{\mathcal{C}} \sum_{Y} Y^{2} P_{t}(\mathcal{C} ; Y)=2 \sum_{\mathcal{C}, \mathcal{C}^{\prime}} K_{\mathrm{I}}\left(\mathcal{C}, \mathcal{C}^{\prime}\right) q_{t}\left(\mathcal{C}^{\prime}\right)+\sum_{\mathcal{C}, \mathcal{C}^{\prime}} K_{1}\left(\mathcal{C}, \mathcal{C}^{\prime}\right) p_{t}\left(\mathcal{C}^{\prime}\right)
\end{aligned}
$$

where the angular brackets denote an average over all configurations and all positions $Y$ at time $t$. In the long-time limit we expect the system to reach a steady state and therefore [23]

$$
p_{t}(\mathcal{C}) \rightarrow p(\mathcal{C}) \quad q_{t}(\mathcal{C}) \rightarrow v t p(\mathcal{C})+r(\mathcal{C})
$$

where from (2), (13), (15) we have

$$
v=\sum_{\mathcal{C}, \mathcal{C}^{\prime}} K_{1}\left(\mathcal{C}, \mathcal{C}^{\prime}\right) p\left(\mathcal{C}^{\prime}\right)
$$

which after evaluation using (1) recovers (3). On substituting (15) into (11), (12) one finds

$$
\begin{gathered}
\sum_{\mathcal{C}^{\prime}}\left[K_{0}\left(\mathcal{C}, \mathcal{C}^{\prime}\right)+K_{1}\left(\mathcal{C}, \mathcal{C}^{\prime}\right)\right] p\left(\mathcal{C}^{\prime}\right)-\sum_{\mathcal{C}^{\prime}}\left[K_{0}\left(\mathcal{C}^{\prime}, \mathcal{C}\right)+K_{1}\left(\mathcal{C}^{\prime}, \mathcal{C}\right)\right] p(\mathcal{C})=0 \\
\sum_{\mathcal{C}^{\prime}}\left[K_{0}\left(\mathcal{C}, \mathcal{C}^{\prime}\right)+K_{1}\left(\mathcal{C}, \mathcal{C}^{\prime}\right)\right] r\left(\mathcal{C}^{\prime}\right)-\sum_{\mathcal{C}^{\prime}}\left[K_{0}\left(\mathcal{C}^{\prime}, \mathcal{C}\right)+K_{1}\left(\mathcal{C}^{\prime}, \mathcal{C}\right)\right] r(\mathcal{C}) \\
=v p(\mathcal{C})-\sum_{\mathcal{C}^{\prime}} K_{1}\left(\mathcal{C}, \mathcal{C}^{\prime}\right) p\left(\mathcal{C}^{\prime}\right)
\end{gathered}
$$

and from (13), (14) that the diffusion constant $\Delta$ is given by

$$
\Delta=\frac{\partial}{\partial t}\left(\left\langle Y_{t}^{2}\right\rangle-\left\langle Y_{t}\right\rangle^{2}\right)=v+2 \sum_{\mathcal{C}, \mathcal{C}^{\prime}} K_{1}\left(\mathcal{C}, \mathcal{C}^{\prime}\right) r\left(\mathcal{C}^{\prime}\right)-2 v \sum_{\mathcal{C}} r(\mathcal{C})
$$

Thus to obtain the diffusion constant one has to solve equations (17), (18). As already argued, the first equation (17) is solved by $p(\mathcal{C})$ equal to a constant and expression (1) gives the correct normalisation. However, the inhomogeneous equation (18) is non-trivial. (Also note that if $r_{0}(\mathcal{C})$ is a particular solution of $(18)$ then the general solution is $r_{0}(\mathcal{C})+\lambda p(\mathcal{C})$, but due to (16) the $\lambda$ dependence disappears from expression (19).

We are now going to show that equation (18) may be sölved by writing $r(\mathcal{C})$ as the trace of a product of semi-infinite non-commuting matrices [11] $B, D, E$

$$
r(\mathcal{C})=R \operatorname{Tr}\left[B \prod_{i=2}^{N} X_{i}\right]
$$


where $X_{i}=D$ if site $i$ is occupied, $X_{i}=E$ if site $i$ is empty, $B$ marks the position of the marked particle (site one) and $R$ is a normalising constant. The translational invariance of the lattice is reflected by the translational invariance of a product of matrices under the trace operation. The matrices $D, E$ satisfy the relation

$$
D E=D+E
$$

and we choose them to be [11]

$$
D=\sum_{i=1}^{\infty}(|i\rangle\langle i|+| i\rangle\langle i+1|) \quad E=\sum_{i=1}^{\infty}(|i\rangle\langle i|+| i+1\rangle\langle i|) .
$$

The matrix $B$ has its first $M$ rows identical to those of $D$ and zeros for all its other elements

$$
B=\sum_{i=1}^{M}(|i\rangle\langle i|+| i\rangle\langle i+1|) \text {. }
$$

In order to prove that equations (20)-(23) solve (18) one has, in principle, one equation of the form (18) to satisfy for each configuration $\mathcal{C}$. However, by an argument similar to that given in [13], which uses repeatedly the identity (21), one can show that any such equation reduces to one of four conditions according to the occupations of sites two and $N$ in configuration $\mathcal{C}$

$$
\begin{array}{ll}
R \operatorname{Tr}\left[\left(E B+B E^{2}-B E-E B E\right) Z_{N-3, M}\right]=(v-1) p(\mathcal{C}) & \text { for } \tau_{2}=0, \tau_{N}=0 \\
R \operatorname{Tr}\left[(D B+B E-D B E) Z_{N-3, M-1}\right]=v p(\mathcal{C}) & \text { for } \tau_{2}=0, \tau_{N}=1 \\
R \operatorname{Tr}\left[(B E D-B D-E B) Z_{N-3, M-1}\right]=(v-1) p(\mathcal{C}) & \text { for } \tau_{2}=1, \tau_{N}=0 \\
R \operatorname{Tr}\left[(B D-D B) Z_{N-3, M-2}\right]=v p(\mathcal{C}) & \text { for } \tau_{2}=1, \tau_{N}=1
\end{array}
$$

where $Z_{N-3, M}$ is a product of $M$ matrices $D$ and $N-3-M$ matrices $E$ in arbitrary order and corresponds to any of the possible configurations for sites $3 \leqslant i \leqslant N-1$. Using the form (22), (23) of $B, D, E$ one can check that the following relations hold:

$$
\begin{aligned}
& E B+B E^{2}-B E-E B E=-|M+1\rangle\langle M| E \\
& D B+B E-D B E=|M\rangle\langle M+1| E . \\
& B E D-B D-E B=-|M+1\rangle\langle M| D \\
& B D-D B=|M\rangle\langle M+1| D .
\end{aligned}
$$

Inserting these expressions into (24) and using the expression (3) for $v$, the conditions (24) become

$$
\begin{aligned}
& R\left\langle M\left|E Z_{N-3, M}\right| M+1\right\rangle=p(\mathcal{C}) M /(N-1) \\
& R\left\langle M+1\left|E Z_{N-3, M-1}\right| M\right\rangle=p(\mathcal{C})(N-1-M) /(N-1) \\
& R\left\langle M\left|D Z_{N-3, M-1}\right| M+1\right\rangle=p(\mathcal{C}) M /(N-1) \\
& R\left\langle M+1\left|D Z_{N-3, M-2}\right| M\right\rangle=p(\mathcal{C})(N-1-M) /(N-1) .
\end{aligned}
$$

In order to show that these conditions can be satisfied, one may use the fact that the matrices $D, E$ are bidiagonal (see 22). The matrix elements of the form $(n\rceil \prod_{i=1}^{L} X_{i}|m\rangle$ can be thought of as representing walks of $L$ steps from site $m$ to site $n$ of a semi-infinite lattice with absorbing boundary at the origin. At step $L+1-i$, if $X_{i}=D$ the walker remains 
at the same site or moves to the left and if $X_{i}=E$ the walker remains at the same site or moves to the right. When there are $K$ matrices $D$ in the product, if $m>K$ the walker cannot reach the origin and the absorbing boundary plays no role. In view of this, the order in which the matrices appear in the matrix elements present in (26) does not matter; thus using the action of $D, E$ on $\langle n|$

$$
\langle n| D=\langle n+1| E=\langle n|+\langle n+1\rangle \quad \text { with }\langle 0\}=0
$$

one obtains

$$
\left\langle n\left|Z_{L, K}\right| m\right\rangle=\left\langle n\left|D^{K} E^{L-K}\right| m\right\rangle=\left\langle n+K\left|E^{L}\right| m\right\rangle=\left(\begin{array}{c}
L \\
K+n-m
\end{array}\right) \quad \text { for } m>K .
$$

With this expression one finds that if the normalisation $R$ is chosen to be

$$
R=p(\mathcal{C})\left[\left(\begin{array}{c}
N-1 \\
M
\end{array}\right)\right]^{-1}=\left[\left(\begin{array}{c}
N-1 \\
M
\end{array}\right)\right]^{-2}
$$

then (26), and therefore (18), are satisfied, hence equation (20) is proven.

The expression of the diffusion constant (19) in terms of matrices is as follows:

$\Delta=v+2\left[\left(\begin{array}{c}N-1 \\ M\end{array}\right)\right]^{-2}\{\operatorname{Tr}[B E G(M, N-2)]-v \operatorname{Tr}[B G(M, N-1)]\}$

where the matrices $G(M, L)$ are given by

$$
G(M, L)=\sum_{\left\{\tau_{k}=1,0 ; 1 \leqslant k \leqslant L\right\}} \delta\left(M-\sum_{j=1}^{L} \tau_{j}\right) \prod_{i=1}^{L}\left(\tau_{i} D+\left(1-\tau_{i}\right) E\right) .
$$

The fact that $B$ has a finite number of non-zero elements means that the traces in (30) are finite sums of matrix elements of $G(M, L)$. These matrix elements can in fact be computed:

$$
\langle n|G(M, L)| m\rangle=\left(\begin{array}{c}
L \\
M
\end{array}\right)\left(\begin{array}{c}
L \\
M+n-m
\end{array}\right)-\left(\begin{array}{c}
L \\
M+n
\end{array}\right)\left(\begin{array}{c}
L \\
M-m
\end{array}\right) .
$$

One way of proving (32) is to use the action (27) of $D, E$ on $\langle n|$ to write a recursion

$$
\begin{aligned}
\langle n|G(M, L)| m\rangle & =\langle n|G(M, L-1)| m\rangle+\langle n-1|G(M, L-1)| m\rangle \\
& +\langle n|G(M-1, L-1)| m\rangle+\langle n+1|G(M-1, L-1)| m\rangle
\end{aligned}
$$

with boundary conditions such that $\langle n|G(M, L)| m\rangle$ vanishes when either $n$ or $m$ is zero. One can then check that (32) satisfies these boundary conditions and the recursion (33). It is possible to evaluate the sums of matrix elements (32) present in (30) by using the identity

$$
\sum_{i=-\infty}^{\infty}\left(\begin{array}{c}
L \\
x+i
\end{array}\right)\left(\begin{array}{c}
L \\
y-i
\end{array}\right)=\left(\begin{array}{c}
2 L \\
x+y
\end{array}\right)
$$

and one finds that

$$
\begin{aligned}
\operatorname{Tr}[B G(M, N-1)]=\sum_{i=1}^{M}[\langle i|+\langle i+1|] G(M, N-1)[i\rangle \\
\quad=\frac{1}{2}\left(\begin{array}{c}
N-1 \\
M
\end{array}\right)\left[\left(\begin{array}{c}
N-1 \\
M+1
\end{array}\right)+(2 M+1)\left(\begin{array}{c}
N \\
M+1
\end{array}\right)\right]-\frac{1}{2}\left(\begin{array}{c}
2 N-1 \\
2 M+1
\end{array}\right)
\end{aligned}
$$




$$
\begin{aligned}
\operatorname{Tr}[B E G(M, N-2)]=\sum_{i=1}^{M}[\langle i-1|+2\langle i|+\langle i+1|] G(M, N-2) \mid i\rangle \\
=\left(\begin{array}{c}
N-2 \\
M
\end{array}\right)\left[\left(\begin{array}{c}
N-1 \\
M+1
\end{array}\right)+M\left(\begin{array}{c}
N \\
M+1
\end{array}\right)\right]-\frac{1}{2}\left(\begin{array}{c}
2 N-2 \\
2 M+1
\end{array}\right) .
\end{aligned}
$$

After a little algebra equation (5) can be obtained from (30) and (35), (36).

In this paper we have presented an exact expression (5) for the diffusion constant of a particle in an asymmetric exclusion process on a ring of size $N$. The results highlight several interesting features of the large $N$ behaviour, namely the scaling for a finite density of particles (8) and the fact that the diffusion constant remains dependent on the particle number in the limit of zero density (6). These features are not present in the expression derived by [18] for an infinite system. (The difference between our result and that of [18] is that here we take $t \rightarrow \infty$ at fixed finite $N$ whereas in [18] $N \rightarrow \infty$ is taken first and then $t \rightarrow \infty$ ).

We believe this work shows that the matrix technique [11] is capable of giving more complicated properties of the steady state than equal-time correlations. It would be of interest to extend our calculations to more general situations such as partially asymmetric exclusion where in time $\mathrm{d} t$ particles can jump to the right with probability $p \mathrm{~d} t$ and to the left with probability $q \mathrm{~d} t$ (in the case $p=q$ the diffusion constant for a finite density of particles in the limit of an infinite system should scale as $N^{-1}[24,25]$ ). One could also look at the two-species problem [13] (at present we have succeeded in calculating the diffusion constant of a single second-class particle in the presence of an arbitrary number of first-class particles). Lastly, it would, of course, be interesting to see whether the matrix approach could be used to obtain more general time correlations in the steady state.

\section{Acknowledgments}

We thank V Hakim, J L Lebowitz and H Spohn for helpful discussions.

\section{References}

[1] Spohn H 1991 Large Scale Dynamics of Interacting Particles (Berlin: Springer)

[2] Katz S, Lebowitz J L and Spohn H 1984 Nonequilibrium steady states of stochastic lattice gas models of fast ionic conductors J. Stat. Phys. 34497

[3] Richards P M 1977 Theory of one-dimensional hopping conductivity and diffusion Phys. Rev. B 161393

[4] Krug J and Spohn H 1991 Kinetic roughening of growing surfaces Solids far from Equilibrium ed C Godrèche (Cambridge: Cambridge University Press)

[5] Kandel D and Mukamel D 1992 Defects, interface profile and phase transitions in growth models Europhys. Lett. 20325

[6] Liggett T M 1985 interacting Particle Systems (Berlin: Springer)

[7] Meakin P, Ramanlal P, Sander L M and Ball R C 1986 Ballistic deposition on surfaces Phys. Rev. A 34 5091

[8] Derrida B, Domany E and Mukamel D 1992 An exact solution of a one dimensional asymmetric exclusion model with open boundaries J. Stat. Phys. 69 667-87

[9] Derrida B and Evans M R 1993 Exact correlation functions in an asymmetric exclusion model with open boundaries J. Physigue I 3311

[10] Schutz G and Domany E 1993 Phase transitions in an exactly soluble one-dimensional exclusion process $J$. Stat. Phys. 72277

[11] Derrida B, Evans M R, Hakim V and Pasquier V 1993 Exact solution of a 1d asymmetric exclusion model using a matrix formulation J. Phys. A: Math. Gen. 261493

[12] Krug J 1991 Boundary-induced phase transitions in driven diffusive systems Phys. Rev. Lett. 671882 
[13] Derrida B, Janowsky S A, Lebowitz J L and Speer E R 1993 Exact solution of the totally asymmetric simple exclusion process: shock profiles $J$. Stat. Phys. in press

[14] Dhar D 1987 An exactly solved model for interfacial growth Phase Transitions 951

[15] Gwa L H and Spohn H 1992 Six-vertex model, roughened surfaces and an asymmetric spin Hamiltonian Phys. Rev. Lett. 68725

[16] Schütz G 1993 Generalized Bethe ansatz solution of a one-dimensional asymmetric exclusion process on a ring with blockage $J$. Stat. Phys. 71471

[17] Kipnis C 1986 Central limit theorems for infinite series of queues and applications to simple exclusion Ann. Prob. 14 397-408

[18] DeMasi and Ferrari P A 1985 Self-diffusion in one-dimensional tattice gases in the presence of an extemal field J. Stat. Phys. 38603

[19] Kutner R and van Beijeren H 1985 J. Stat. Phys. 39317

[20] van Beijeren $H 1991$ Fluctuations in the motions of mass and of patterns in one-dimensional driven diffusive systems $J$. Stat. Phys. 6347

[21] Majumdar S N and Barma M 1991 Tag diffusion in driven systems, growing interfaces, and anomalous fluctuations Phys. Rev. B 445306

[22] Kardar M, Parisi G and Zhang Y-C 1986 Phys. Rev. Lett. 56889

[23] Derrida B 1983 Velocity and diffusion constant of a periodic one-dimensional hopping model J. Stat. Phys. 31 433-50

[24] van Beijeren H, Kehr K W and Kutner R 1983 Diffusion in concentrated lattice gases III. Tracer diffusion on a one-dimensional lattice Phys. Rev. B 285711

[25] Ferrari P A, Goldstein S and Lebowitz J I 1985 Diffusion, mobility and the Einstein relation Statistical Physic's and Dynamical Systems ed J Fritz, A Jaffe and D Szász (Boston, MA: Birkhäuser) 